\title{
ANNALES
}

UNIVERSITATIS MARIAE CURIE-SKŁODOWSKA

L UBLIN - POLONIA

\section{Majorization for certain classes of meromorphic functions defined by integral operator}

\begin{abstract}
Here we investigate a majorization problem involving starlike meromorphic functions of complex order belonging to a certain subclass of meromorphic univalent functions defined by an integral operator introduced recently by Lashin.
\end{abstract}

1. Introduction and preliminaries. Let $f(z)$ and $g(z)$ be analytic in the open unit disk

$$
\triangle=\{z \in \mathbb{C} \text { and }|\mathrm{z}|<1\} .
$$

For analytic functions $f(z)$ and $g(z)$ in $\Delta$, we say that $f(z)$ is majorized by $g(z)$ in $\Delta$ (see [9]) and write

$$
f(z) \ll g(z) \quad(z \in \Delta),
$$

if there exists a function $\phi(z)$, analytic in $\Delta$ such that $|\phi(z)| \leq 1$, and

$$
f(z)=\phi(z) g(z)(z \in \Delta) .
$$

Let $\Sigma$ denote the class of meromorphic functions of the form

$$
f(z)=\frac{1}{z}+\sum_{k=1}^{\infty} a_{k} z^{k},
$$

2000 Mathematics Subject Classification. Primary 30C45; Secondary 30C80.

Key words and phrases. Meromorphic univalent functions, majorization property, starlike functions, integral operators. 
which are analytic and univalent in the punctured unit disk

$$
\Delta^{*}:=\{z \in \mathbb{C}: 0<|z|<1\}:=\Delta \backslash\{0\}
$$

with a simple pole at the origin.

For functions $f_{j} \in \Sigma$ given by

$$
f_{j}(z)=\frac{1}{z}+\sum_{k=1}^{\infty} a_{k, j} z^{k} \quad\left(j=1,2 ; z \in \Delta^{*}\right),
$$

we define the Hadamard product (or convolution) of $f_{1}$ and $f_{2}$ by

$$
\left(f_{1} * f_{2}\right)(z)=\frac{1}{z}+\sum_{k=1}^{\infty} a_{k, 1} a_{k, 2} z^{k}=\left(f_{2} * f_{1}\right)(z) .
$$

Analogously to the operators defined by Jung, Kim and Srivastava [7] on the normalized analytic functions, Lashin [8] introduced the following integral operators

$$
\mathcal{P}_{\beta}^{\alpha}: \Sigma \longrightarrow \Sigma
$$

defined by

$$
\mathcal{P}_{\beta}^{\alpha}=\mathcal{P}_{\beta}^{\alpha} f(z)=\frac{\beta^{\alpha}}{\Gamma(\alpha)} \frac{1}{z^{\beta+1}} \int_{0}^{z} t^{\beta}\left(\log \frac{z}{t}\right)^{\alpha-1} f(t) d t
$$

$\left(\alpha>0, \beta>0 ; z \in \Delta^{*}\right)$, where $\Gamma(\alpha)$ is the familiar Gamma function.

Using the integral representation of the Gamma function and (1.4), it can be easily shown that

$$
\mathcal{P}_{\beta}^{\alpha} f(z)=\frac{1}{z}+\sum_{k=1}^{\infty}\left(\frac{\beta}{\beta+k+1}\right)^{\alpha} a_{k} z^{k}, \quad\left(\alpha>0, \beta>0 ; z \in \Delta^{*}\right) .
$$

Obviously

$$
\mathcal{P}_{\beta}^{1} f(z):=\mathcal{J}_{\beta}
$$

The operator

$$
\mathcal{J}_{\beta}: \Sigma \longrightarrow \Sigma
$$

has also been studied by Lashin [8].

It is easy to verify that (see [8]),

$$
z\left(\mathcal{P}_{\beta}^{\alpha} f(z)\right)^{\prime}=\beta \mathcal{P}_{\beta}^{\alpha-1} f(z)-(\beta+1) \mathcal{P}_{\beta}^{\alpha} f(z) .
$$

Definition 1.1. A function $f(z) \in \Sigma$ is said to be in the class $\mathcal{S}_{\beta}^{\alpha, j}(\gamma)$ of meromorphic functions of complex order $\gamma \neq 0$ in $\Delta$ if and only if

$$
\Re\left\{1-\frac{1}{\gamma}\left(\frac{z\left(\mathcal{P}_{\beta}^{\alpha} f(z)\right)^{(j+1)}}{\left(\mathcal{P}_{\beta}^{\alpha} f(z)\right)^{(j)}}+j+1\right)\right\}>0
$$

$\left(z \in \Delta, j \in \mathbb{N}_{0}=\mathbb{N} \cup\{0\}, \alpha>0, \beta>0, \gamma \in \mathbb{C} \backslash\{0\}\right)$. 
Clearly, we have the following relationships:

$$
\begin{aligned}
& \text { (i) } \mathcal{S}_{\beta}^{0,0}(\gamma)=\mathcal{S}(\gamma) \quad(\gamma \in \mathbb{C} \backslash\{0\}), \\
& \text { (ii) } \mathcal{S}_{\beta}^{0,0}(1-\eta)=\mathcal{S}^{*}(\eta) \quad(0 \leq \eta<1) .
\end{aligned}
$$

The classes $\mathcal{S}(\gamma)$ and $\mathcal{S}^{*}(\eta)$ are said to be classes of meromorphic starlike univalent functions of complex order $\gamma \neq 0$ and meromorphic starlike univalent functions of order $\eta(\eta \in \Re$ such that $0 \leq \eta<1)$ in $\Delta^{*}$.

A majorization problem for the normalized classes of starlike functions has been investigated by Altinas et al. [1] and MacGregor [9]. In the recent paper Goyal and Goswami [2] generalized these results for the class of multivalent functions, using fractional derivatives operators. Further, Goyal et al. [3], Goswami and Wang [4], Goswami [5], Goswami et al. [6] studied majorization property for different classes. In this paper, we will study majorization properties for the class of meromorphic functions using integral operator $\mathcal{P}_{\beta}^{\alpha}$.

\section{Majorization problems for the class $\mathcal{S}_{\beta}^{\alpha, j}(\gamma)$.}

Theorem 2.1. Let the function $f \in \Sigma$ and suppose that $g \in \mathcal{S}_{\beta}^{\alpha, j}(\gamma)$. If $\left(\mathcal{P}_{\beta}^{\alpha} f(z)\right)^{(j)}$ is majorized by $\left(\mathcal{P}_{\beta}^{\alpha} g(z)\right)^{(j)}$ in $\Delta^{*}$, then

$$
\left|\left(\mathcal{P}_{\beta}^{\alpha-1} f(z)\right)^{(j)}\right| \leq\left|\left(\mathcal{P}_{\beta}^{\alpha-1} g(z)\right)^{(j)}\right| \text { for }|z| \leq r_{1}(\beta, \gamma),
$$

where

$$
r_{1}(\beta, \gamma)=\frac{k_{1}-\sqrt{k_{1}^{2}-4 \beta|\beta+2 \gamma|}}{2|\beta+2 \gamma|}
$$

and

$$
k_{1}=\beta+2+|\beta+2 \gamma|,\left(\beta>0, j \in \mathbb{N}_{0}, \gamma \in \mathbb{C} \backslash\{0\}\right) .
$$

Proof. Since $g \in \mathcal{S}_{\beta}^{\alpha, j}(\gamma)$, we find from (2.1) that if

$$
h_{1}(z)=1-\frac{1}{\gamma}\left(\frac{z\left(\mathcal{P}_{\beta}^{\alpha} g(z)\right)^{(j+1)}}{\left(\mathcal{P}_{\beta}^{\alpha} g(z)\right)^{(j)}}+j+1\right)
$$

$\left(\alpha, \beta>0, \gamma \in \mathbb{C} \backslash\{0\}, j \in \mathbb{N}_{0}\right)$, then $\Re\left\{h_{1}(z)\right\}>0(z \in \Delta)$ and

$$
h_{1}(z)=\frac{1+w(z)}{1-w(z)} \quad(w \in \mathcal{P}),
$$

where $\mathcal{P}$ denotes the well-known class of bounded analytic functions in $\Delta$ and $w(z)=c_{1} z+c_{2} z^{2}+\ldots$ satisfies the conditions

$$
w(0)=0 \text { and }|w(z)| \leq|z|(z \in \Delta) .
$$


Making use of (2.3) and (2.4), we get

$$
\frac{z\left(\mathcal{P}_{\beta}^{\alpha} g(z)\right)^{(j+1)}}{\left(\mathcal{P}_{\beta}^{\alpha} g(z)\right)^{(j)}}=\frac{(1+j-2 \gamma) w(z)-(1+j)}{1-w(z)} .
$$

By the principle of mathematical induction, and (1.11), we easily get

$$
z\left(\mathcal{P}_{\beta}^{\alpha} g(z)\right)^{(j+1)}=\beta\left(\mathcal{P}_{\beta}^{\alpha-1} g(z)\right)^{(j)}-(\beta+j+1)\left(\mathcal{P}_{\beta}^{\alpha} g(z)\right)^{(j)},
$$

$\left(\alpha>1, \beta>0 ; z \in \Delta^{*}\right)$. Now using (2.6) in (2.5), we find that

$$
\begin{aligned}
\frac{\beta\left(\mathcal{P}_{\beta}^{\alpha-1} g(z)\right)^{(j)}}{\left(\mathcal{P}_{\beta}^{\alpha} g(z)\right)^{(j)}} & =(\beta+j+1)+\frac{(1+j-2 \gamma) w(z)-(1+j)}{1-w(z)} \\
& =\frac{\beta-(\beta+2 \gamma) w(z)}{1-w(z)}
\end{aligned}
$$

or

$$
\left(\mathcal{P}_{\beta}^{\alpha} g(z)\right)^{(j)}=\frac{\beta(1-w(z))}{\beta-(\beta+2 \gamma) w(z)}\left(\mathcal{P}_{\beta}^{\alpha-1} g(z)\right)^{(j)} .
$$

Since $|w(z)| \leq|z|(z \in \Delta)$, the formula (2.6) yields

$$
\left|\left(\mathcal{P}_{\beta}^{\alpha} g(z)\right)^{(j)}\right| \leq \frac{\beta[1+|z|]}{\beta-|\beta+2 \gamma||z|}\left|\left(\mathcal{P}_{\beta}^{\alpha-1} g(z)\right)^{(j)}\right| .
$$

Next since $\left(\mathcal{P}_{\beta}^{\alpha} f(z)\right)^{(j)}$ is majorized by $\left(\mathcal{P}_{\beta}^{\alpha} g(z)\right)^{(j)}$ in the unit disk $\Delta^{*}$, from (1.3), we have

$$
\left(\mathcal{P}_{\beta}^{\alpha} f(z)\right)^{(j)}=\phi(z)\left(\mathcal{P}_{\beta}^{\alpha} g(z)\right)^{(j)} .
$$

Differentiating it with respect to $z$ and multiplying by $z$, we get

$$
z\left(\mathcal{P}_{\beta}^{\alpha} f(z)\right)^{(j+1)}=z \varphi^{\prime}(z)\left(\mathcal{P}_{\beta}^{\alpha} g(z)\right)^{(j)}+z \varphi(z)\left(\mathcal{P}_{\beta}^{\alpha} g(z)\right)^{(j+1)} .
$$

Using (2.7), in the above equality, it yields

$$
\left(\mathcal{P}_{\beta}^{\alpha-1} f(z)\right)^{(j)}=\frac{z \varphi^{\prime}(z)}{\beta}\left(\mathcal{P}_{\beta}^{\alpha} g(z)\right)^{(j)}+\varphi(z)\left(\mathcal{P}_{\beta}^{\alpha-1} g(z)\right)^{(j)} .
$$

Thus, nothing that $\varphi \in \mathcal{P}$ satisfies the inequality (see, e.g. Nehari [6])

$$
\left|\varphi^{\prime}(z)\right| \leq \frac{1-|\varphi(z)|^{2}}{1-|z|^{2}}
$$

and making use of (2.8) and (2.10) in (2.9), we get

$$
\begin{aligned}
& \left|\left(\mathcal{P}_{\beta}^{\alpha-1} f(z)\right)^{(j)}\right| \\
& \quad \leq\left(|\varphi(z)|+\frac{1-|\varphi(z)|^{2}}{1-|z|} \frac{|z|}{[\beta-|2 \gamma+\beta||z|]}\right)\left|\left(\mathcal{P}_{\beta}^{\alpha-1} g(z)\right)^{(j)}\right|,
\end{aligned}
$$

which upon setting

$$
|z|=r \text { and }|\varphi(z)|=\rho \quad(0 \leq \rho \leq 1)
$$


leads us to the inequality

$$
\left|\left(\left(\mathcal{P}_{\beta}^{\alpha-1} f(z)\right)^{(j)}\right)\right| \leq \frac{\Theta(\rho)}{(1-r)(\beta-|2 \gamma+\beta| r)}\left|\left(\mathcal{P}_{\beta}^{\alpha-1} g(z)\right)^{(j)}\right|,
$$

where

$$
\Theta(\rho)=-r \rho^{2}+(1-r)(\beta-|2 \gamma+\beta| r) \rho+r
$$

takes its maximum value at $\rho=1$, with $r_{2}=r_{2}(\beta, \gamma)$, where $r_{2}(\beta, \gamma)$ is given by equation (2.2). Furthermore, if $0 \leq \rho \leq r_{2}(\beta, \gamma)$, then the function $\theta(\rho)$ defined by

$$
\theta(\rho)=-\sigma \rho^{2}+(1-\sigma)(\beta-|2 \gamma+\beta| \sigma) \rho+\sigma
$$

is an increasing function on the interval $0 \leq \rho \leq 1$, so that

$$
\theta(\rho) \leq \theta(1)=(1-\sigma)(\beta-|2 \gamma+\beta| \sigma),
$$

$\left(0 \leq \rho \leq 1 ; 0 \leq \sigma \leq r_{1}(\beta, \gamma)\right)$. Hence upon setting $\rho=1$ in (2.14), we conclude that (2.1) of Theorem 2.1 holds true for $|z| \leq r_{1}(\beta, \gamma)$, where $r_{1}(\beta, \gamma)$ is given by $(2.2)$. This completes the proof of Theorem 2.1.

Setting $\alpha=1$ in Theorem 2.1, we get

Corollary 2.1. Let the function $f \in \Sigma$ and suppose that $g \in \mathcal{S}_{\beta}^{1, j}(\gamma)$. If $\left(\mathcal{J}_{\beta} f(z)\right)^{(j)}$ is majorized by $\left(\mathcal{J}_{\beta} g(z)\right)^{(j)}$ in $\Delta^{*}$, then

$$
\left|(f(z))^{(j)}\right| \leq\left|(g(z))^{(j)}\right| \text { for }|z| \leq r_{2}(\beta, \gamma),
$$

where

and

$$
r_{2}(\beta, \gamma)=\frac{k_{2}-\sqrt{k_{2}^{2}-4 \beta|\beta+2 \gamma|}}{2|\beta+2 \gamma|}
$$

$$
k_{2}=\beta+2+|\beta+2 \gamma|, \quad\left(\beta>0, j \in \mathbb{N}_{0}, \gamma \in \mathbb{C} \backslash\{0\}\right) .
$$

Further putting $\beta=1$ and $\gamma=1-\eta, j=0$ in Corollary 2.1, we get

Corollary 2.2. Let the function $f \in \Sigma$ and suppose that $g \in \mathcal{S}_{1}^{1,0}(1-\eta)$. If $\left(\mathcal{J}_{1} f(z)\right)$ is majorized by $\left(\mathcal{J}_{1} g(z)\right)$ in $\Delta^{*}$, then

$$
|f(z)| \leq|g(z)| \text { for }|z| \leq r_{3}
$$

where

$$
r_{3}=\frac{3-\eta-\sqrt{\eta^{2}-4 \eta+6}}{3-\eta}
$$

For $\eta=0$, the above corollary reduces to the following result:

Corollary 2.3. Let the function $f(z) \in \Sigma$ and suppose that $g \in \mathcal{S}_{1}^{1,0}(1):=$ $\mathcal{S}_{1}^{1,0}$. If $\left(\mathcal{J}_{1} f(z)\right)$ is majorized by $\left(\mathcal{J}_{1} g(z)\right)$ in $\Delta^{*}$, then

$$
|f(z)| \leq|g(z)| \text { for }|z| \leq \frac{3-\sqrt{6}}{3} .
$$


Acknowledgments. The first author is thankful to CSIR, New Delhi, India for awarding Emeritus Scientist under scheme No. 21(084)/10/EMR-II.

\section{REFERENCES}

[1] Altintaş, O., Özkan, Ö., Srivastava, H. M., Majorization by starlike functions of complex order, Complex Variables Theory Appl. 46 (2001), 207-218.

[2] Goyal, S. P., Goswami, P., Majorization for certain classes of analytic functions defined by fractional derivatives, Appl. Math. Lett. 22 (12) (2009), 1855-1858.

[3] Goyal, S. P., Bansal S. K., Goswami, P., Majorization for certain classes of analytic functions defined by linear operator using differential subordination, J. Appl. Math. Stat. Inform. 6 (2) (2010), 45-50.

[4] Goswami, P., Wang, Z.-G., Majorization for certain classes of analytic functions, Acta Univ. Apulensis Math. Inform. 21 (2009), 97-104.

[5] Goswami, P., Aouf, M. K., Majorization properties for certain classes of analytic functions using the Sălăgean operator, Appl. Math. Lett. 23 (11) (2010), 1351-1354.

[6] Goswami, P., Sharma, B., Bulboacă, T., Majorization for certain classes of analytic functions using multiplier transformation, Appl. Math. Lett. 23 (10) (2010), 633-637.

[7] Jung, I. B., Kim, Y. C., Srivastava, H. M., The Hardy space of analytic functions associated with certain one-parameter families of integral operator, J. Math. Anal. Appl. 176 (1) (1993), 138-147.

[8] Lashin, A. Y., On certain subclasses of meromorphic functions associated with certain integral operators, Comput. Math. Appl., 59 (1) (2010), 524-531.

[9] MacGreogor, T. H., Majorization by univalent functions, Duke Math. J. 34 (1967), 95-102.

[10] Nehari, Z., Conformal Mapping, MacGraw-Hill Book Company, New York, Toronto and London, 1955.

\section{S. P. Goyal}

Department of Mathematics

University of Rajasthan

Jaipur-302055

India

e-mail: somprg@gmail.com
Pranay Goswami

Department of Mathematics

AMITY University Rajasthan

Jaipur-302002

India

e-mail: pranaygoswami83@gmail.com

Received March 7, 2011 